\title{
Application of Infrared CCD Thermal Imaging in the Measurement of the Temperature of Aluminum Electrolyte
}

\author{
Zhengguang Xu, Shuai Zhang ${ }^{*}$, Jinjun Wang \\ School of Automation and Electrical Engineering, University of Science and Technology Beijing, Beijing, 100083, China \\ ${ }^{*}$ Corresponding author
}

\begin{abstract}
In this paper, a method that non-contact measurement of the temperature is proposed. It is based on the principle of infrared thermal radiation and using the infrared CCD thermal imaging technology. In order to measure the temperature of aluminum electrolyte, an infrared image temperature measurement system is designed. The system consists of hardware and software. The section of hardware mainly function is system control and video capture and the section of software is mainly used to process the image that from the video. Through experimental data we can fit the curve which can express the relationship between the gray value and temperature of aluminum electrolyte. Finally, read the gray value of aluminum electrolyte image and put it into the calibration curve, and calculate the temperature of the aluminum electrolyte. Experimental results show that the system is easy to use, and can accurately measure the temperature of aluminum electrolyte.
\end{abstract}

Keywords-aluminum electroyte; infrared CCD ; non-contact measurment; temperature calibration

\section{INTRODUCTION}

At present, the temperature of the aluminum electrolyte is measured by the method of artificial temperature measurement. The measuring method is to insert the probe into the electrolyte, and the temperature of the electrolyte can be read when the heat balance is reached. However, this method requires the thermocouple to be inserted into the electrolyte in the molten state, which not only increases the labor intensity of workers, but also erode the thermocouple, which leads to the shortened life span of the thermocouple. In order to extend the life of the thermocouple, Yu Xuebin designed a thermocouple [1] with heat insulation and high temperature resistance between the thermocouple wire and the protective sleeve. Based on the heat transfer mechanism, Yuan Quan and Wang Huazhang [2], carried out a lot of experiments, and established the model of temperature through mathematical analysis and processing. According to the model, the temperature of the electrolyte is calculated when the temperature of the electrolyte and thermocouple still far away from the equilibrium. Thus, the contact time of the thermocouple and the electrolyte is shortened, and improved the thermocouple life. However, these measurement methods are artificial, the measured data into the computer one by one, for the control of the real-time control of the slot machine. This not only increases the labor intensity of workers, but also prone to error measurement.
However, in the aluminum electrolysis, the on-line detection of non-contact temperature is seldom used, because of the serious electromagnetic interference and the large dust in the electrolytic workshop. It is well known that the infrared image can reflect the surface temperature of the object and the temperature of the aluminum electrolyte is high, so the temperature of the aluminum electrolyte can be measured by the infrared image. Renier.E [3] designed a set of detection device which use multiple CCD camera real-time monitoring the process of welding and set up a temperature field that based on the CCD camera. Xu Zhengguang and Shu Yun designed an infrared CCD thermal imaging device [4], and established the temperature field mode of heating furnace. As well as we know that infrared CCD can receive the energy of the radiation of the object, and convert the energy into the image signal. Therefore, we can use the infrared image of the aluminum electrolyte to measure its temperature.

In this paper, a set of aluminum electrolyte temperature on line-detection system is designed, which can measure the temperature of the aluminum electrolyte through the infrared image.

\section{INFRARED IMAGE TEMPERATURE MEASUREMENT SYSTEM}

Infrared image temperature measurement system consists of hardware and software. The section of hardware mainly function is system control and video capture, which mainly include computer, infrared camera, driving mechanism, control cabinet and remote control terminal. The section of software is mainly used to process the image that from the video and calculate the temperature of aluminum electrolyte.

\section{A. Infrared Image Acquisition Device}

The infrared image acquisition device is installed on the aluminum electrolysis cell, and the schematic diagram is shown in FIGURE I. 


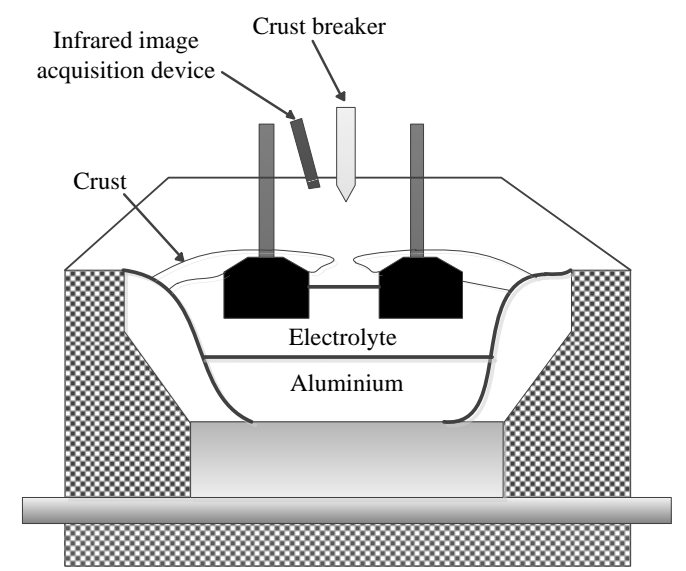

FIGURE I. SCHEMATIC DIAGRAM OF ALUMINUM ELECTROLYTIC CELL.

The infrared image acquisition device mainly comprises a driving mechanism, a camera protection probe and an infrared camera. Driving mechanism drives chain transmission through electric motor, which make the protection probe c move along the slider rail. The infrared image acquisition device schematic diagram shown in FIGURE II.

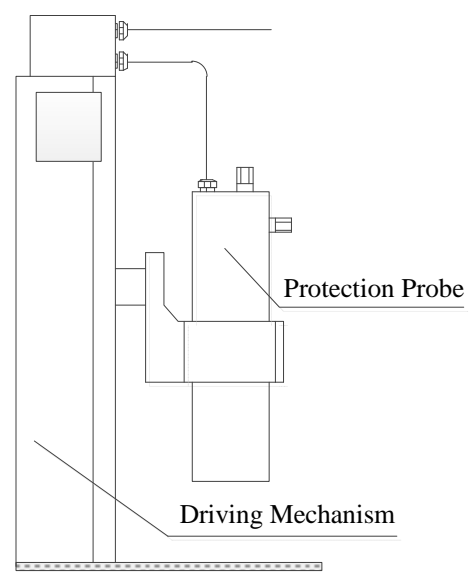

FIGURE II. INFRARED IMAGE ACQUISITION DEVICE SCHEMATIC DIAGRAM

The control cabinet can control the driving mechanism to drive the protection probe (infrared camera) to move up and down, which is convenient for observing the infrared image of the aluminum electrolyte in the electrolytic cell. When the temperature which in the protection probe is too high, the control system can control the driving mechanism to drive the protective probe to move away from the aluminum electrolyte to protect the camera equipment in the probe. As the aluminum electrolyte temperature is high, in order to prevent the temperature that in the protection probe is too high. There are two cooling gas interfaces on the protection probe, where the gas from the side interface has the function of cleaning the lens. The cooling gas passes through the interlayer and is discharged from the front end of the probe, which form air curtain and protect lens from pollution. The cooling gas interface of the back cover of the probe is to enter the cooling gas after filtering through the gas processing device in the electric control cabinet, which play a role of cooling the camera.

\section{B. CCD Imaging Device}

The CCD imaging device is installed in the inner of the protection probe, which mainly includes an infrared filter lens, a wide-angle pinhole lens and a CCD camera device. The infrared filter lens is connected to the front end of the wide angle pinhole lens through the thread. The wide-angle pinhole lens is in front of 6 lenses which are divided into two groups. Where the function of the first three lenses is imaging and the last three functions is to adjust the focal length. That makes the high temperature aluminum electrolyte imaging on the target surface of CCD. Next, the CCD camera device converts the optical signal into electrical signals, which is connected with the display through a video output terminal. Finally the aluminum electrolyte image is displayed on the display device. The schematic diagram of the internal structure of the protection probe is shown in FIGURE III.

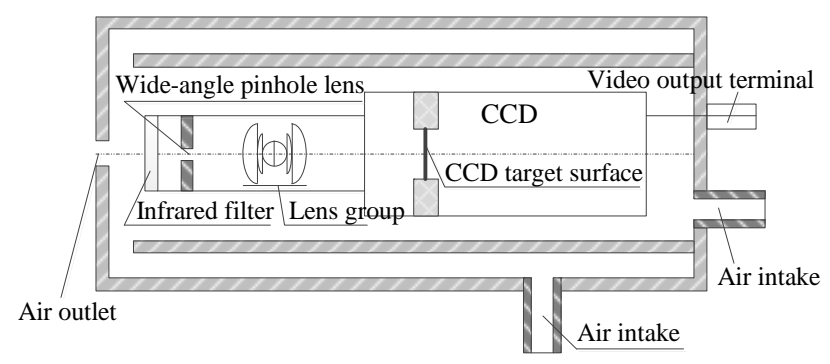

FIGURE III. SCHEMATIC DIAGRAM OF THE INTERNAL STRUCTURE OF THE PROTECTION PROBE

Infrared filter can remove the interference of visible light and the infrared which do not need, in order to improve the contrast of the image. Wide-angle lens video angle is $2 w \geq 110^{\circ}$.

\section{Infrared Image Temperature Measurement Principle}

Because there is a lot of dust in the aluminum electrolytic cell, the visible light of electrolyte radiation occur scattering phenomenon. It not only reduces the intensity of the light whose project on the CCD target surface, but also increase the intensity of the light which is internal of the electrolytic cell. It is equivalent to the effect that between the aluminum electrolyte and CCD target surface have a layer of bright yarn mantle. Thus greatly reduces the contrast of different parts of the aluminum electrolyte image. According to the infrared physics we can know that the longer the wavelength the greater the ability to penetrate the turbid medium. Because the advantage that the infrared ray wavelength is more than the visible light and the scattering is little, it is reasonable to use infrared image in the industrial field.

The temperature of the electrolyte in the aluminum electrolytic cell is in the range of $900^{\circ} \mathrm{C}-1000^{\circ} \mathrm{C}$. Therefore, the electrolyte can radiate all kinds of visible light and infrared light at different wavelengths. The role of the CCD target surface is the same as the retina of the human eye. However, the range of the response wavelength [5], [6] of CCD target surface is wider than that of the human eye. It can be extended 
to $1.1 \mu$. The spectral characteristics of the CCD target surface are shown in FIGURE IV.

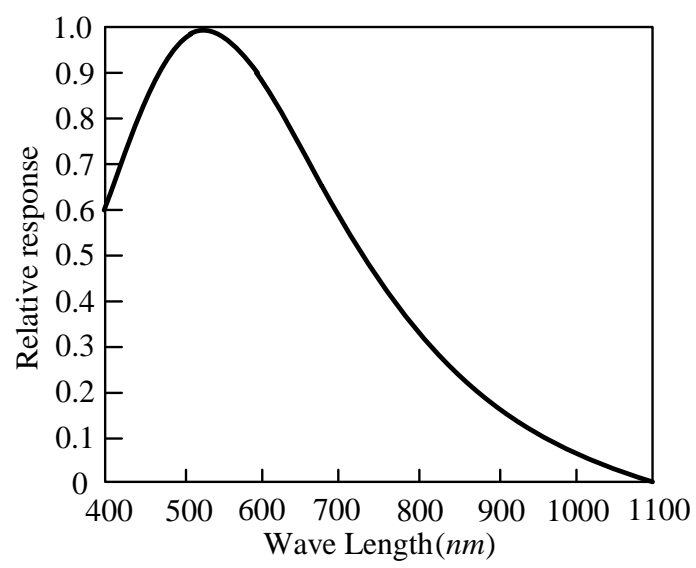

FIGURE IV. THE SPECTRAL CHARACTERISTICS OF THE CCD TARGET SURFACE

Only the radiation characteristics of the object are consistent with the spectral characteristics of the CCD target surface and the amount of radiation energy is appropriate to produce the best image effect. The curve of the black body radiation is shown in FIGURE V .

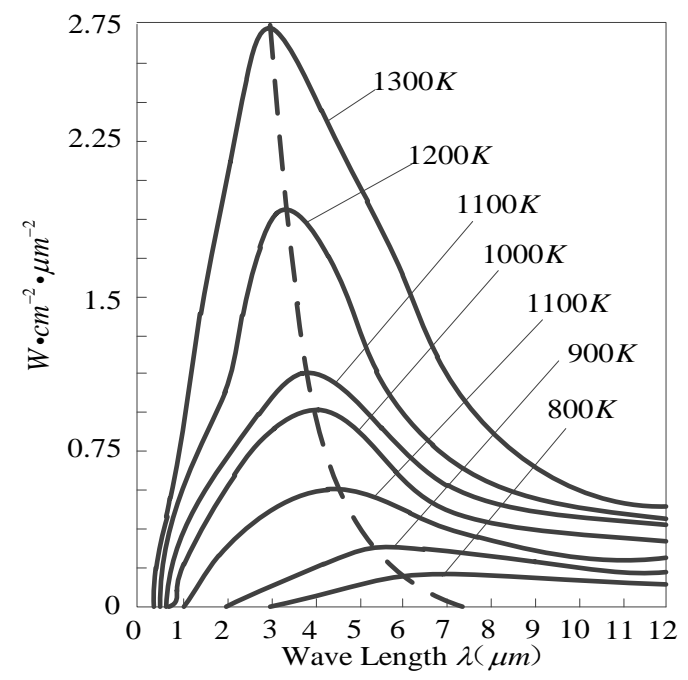

FIGURE V. THE CURVE OF BLACK BODY RADIATION.

According to Wien's displacement law [7], [8], under a certain temperature, the product of the absolute black body temperature and the wavelength which correspond to the maximum value of the radiation energy is a constant, namely

$$
\lambda \cdot T=b
$$

Where $b=0.0028977 m \cdot K$ is the Wien constant. The radiation wavelength of the $950^{\circ} \mathrm{C}$ temperature electrolyte is $\lambda_{m}=2.369 \mu$. According to the Stefan-Boltzmann formula to describe the curve showed that the electrolyte between $0.76 \mu$ and $1.1 \mu$ still has a very strong radiation. So we choose a narrow band filter with a wavelength of $\Delta \lambda=0.8 \mu-0.9 \mu$. The relative response of the filter is shown in FIGURE VI.

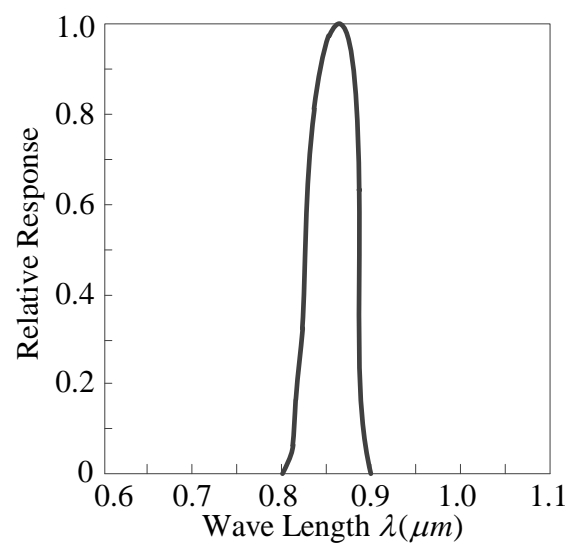

FIGURE VI. THE RELATIVE RESPONSE OF NARROW BAND FILTER.

Placed infrared filters at the front of the lens will remove visible light and infrared light which do not need. The remaining infrared light is focused on the CCD target surface through the optical lens. Then the infrared image of the electrolyte is displayed on the display through the CCD circuit. As shown in FIGURE VII.

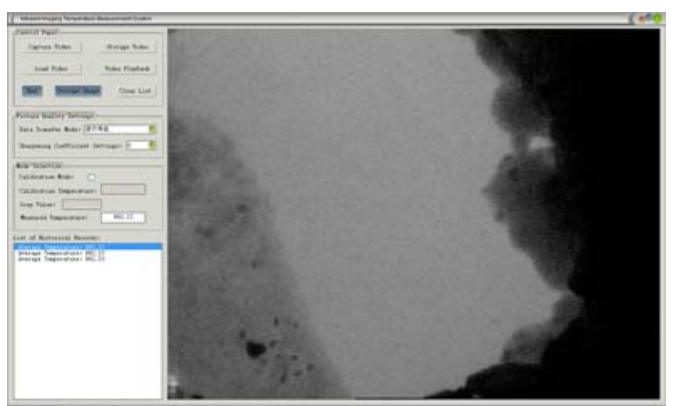

FIGURE VII. INFRARED IMAGE OF ALUMINUM ELECTROLYTE.

\section{TEMPERATURE CALIBRATION}

Because the aluminum electrolyte is covered with a layer of crust over time on the surface of the electrolyte, crust breaker must break the crust to expose the electrolyte for reading the gray level of it image. There is a pneumatic crust breaker on the electrolytic cell, as shown in FUGURE I. When the switch is triggered, the crust breaker will move downwards from the upper and break the crust to expose the electrolyte. Therefore, the image acquisition system will capture the image of the electrolyte and read its gray value.

The Stefan-Boltzmann law shows that the infrared image which captured by infrared CCD camera is the result of a series of conversion of aluminum electrolytic radiation power. So the gray value of the image of aluminum electrolyte has a certain relationship with its temperature. Therefore, it can use the gray value of the infrared image of aluminum electrolyte to calculate its temperature. We used the experimental method to find the 
relationship between the image gray value and the temperature of aluminum electrolyte. The scatter diagram of the gray value and the temperature values is shown in FIGURE VIII.

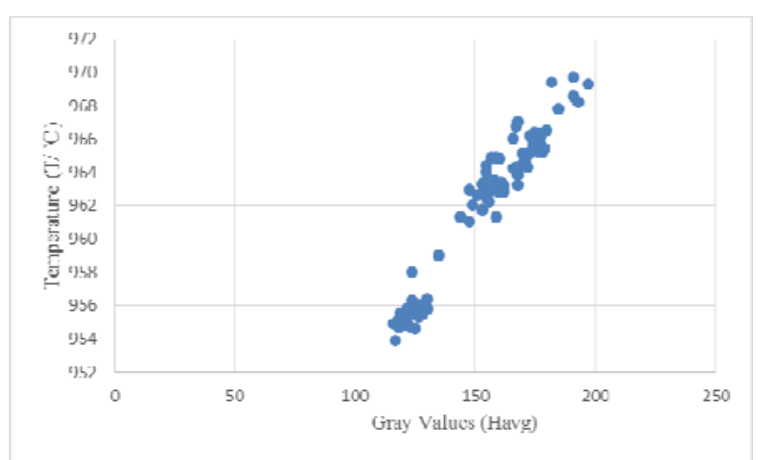

FIGURE VIII. THE SCATTER DIAGRAM OF GRAY VALUES AND TEMPERATURE VALUES

We can see that the gray value of the aluminum electrolytic image has a linear relationship with the temperature of the aluminum electrolyte. Through these data we can fit the curve which can express the relationship between the gray value and temperature of aluminum electrolyte. As shown in FIGURE IX.

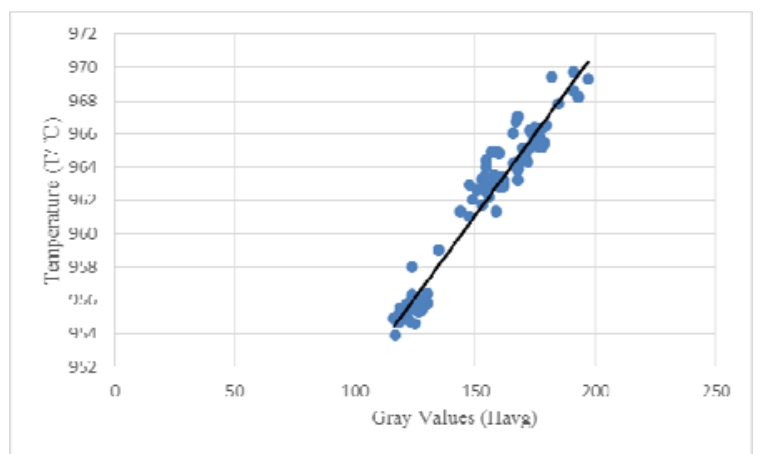

FIGURE IX. RELATIONSHIP BETWEEN GRAY VALUE AND TEMPERATURE VALUE

The expression of the curve is

$$
T=0.1977 \cdot \operatorname{Havg}+931.5
$$

Temperature measurement system will read a gray value when the crust breaker breaks the crust and letting the aluminum electrolyte expose. Then according to (2) calculate the temperature of aluminum electrolyte and display it in the interface, as shown in FIGURE VII.

\section{CONCLUSION}

In order to measure the temperature of aluminum electrolyte, a set of infrared image temperature measurement system is designed. The electric control system of the hardware system ensures the normal operation of the system, even if the unexpected situation also can ensure that the system is not damaged. Through the analysis of the principle of infrared image temperature measurement and experimental demonstration, we found the relationship between the gray value of the aluminum electrolytic image and its temperature. Finally, the gray value of the aluminum electrolyte is read by the method of image processing, and the temperature of the electrolyte is calculated. The experimental results show that the temperature error of using the method of the temperature measurement system and thermocouple measure aluminum electrolyte temperature is $\pm 5^{\circ} \mathrm{C}$, which in the range of error allowed.

\section{REFERENCES}

[1] Yu Xuebin, Wang Huazhang, Xue Jian, Liu Yexiang, "Thermocouple for Measurement of Temperature in Aluminum Cells by Dynamic method,” Nonferrous Metals, Vol.2, pp. 76-79, 1996.

[2] Yuan Quan, Wang Huazhang, Liu Yexiang, "Study On the Temperature of Aluminum Electrolyte by Dynamic Method,” Light Metals, Vlo.7, pp. 32-33, 1993.

[3] Renier. E, Meriaudeau. F, Truchetet. F, "CCD Technology Applied to Industrial Welding Applications," Proceedings of the 1996 8th Mediterranean Electrotechnical Conference, MELECON'06. Part 3(of 3), Vol.3, pp. 1335-1338, 1996.

[4] Xu Zhengguang, Shu Yun, Zhang Lixin, Fu Peizhong, "Study on Temperature Field Model of Slab Based On Infrared CCD," Metallurgical Industry Automation, Vol.1,pp. 24-27, 2005.

[5] Gang Xiao, Kaikai Guo, Weiping Xu, Mingjiang Ni, Zhongyang Luo, Kefa Cen, "An Improved Method of Lambertian CCD-camera Radiation Flux Measurement Based on SMARTS to Reduce Spectral Errors," Energy, Vol.67, pp. 74-80, 2014.

[6] Yuheng Zhang, Yihua Yan, "Design of Frame-transferred Surface Array CCD Imaging System for Dark Objects," Chinese Astronomy and Astrophysics, Vol. 40, pp. 129-140, 2016.

[7] Stewart, Seán M, "Spectral Peaks and Wien's Displacement Law," Thermophysics and Heat Transfer, Vol. 26, pp. 689-691, 2012.

[8] De, Lima, J. A. S, Santos, J M, "Generalized Stefan Boltzmann Law," International Journal of Theoretical Physics, Vol. 34, pp. 127, 1995. 